\title{
Whooping cough in school age children presenting with persistent cough in UK primary care after introduction of the preschool pertussis booster vaccination: prospective cohort study
}

\author{
(c) (1) $(9)$ OPEN ACCESS
}

\author{
Kay Wang academic clinical lecturer ${ }^{1}$, Norman K Fry deputy head ${ }^{2}$, Helen Campbell senior clinical \\ scientist $^{3}$, Gayatri Amirthalingam consultant epidemiologist ${ }^{3}$, Timothy G Harrison head ${ }^{2}$, David Mant \\ emeritus professor of general practice ${ }^{1}$, Anthony Harnden associate professor ${ }^{1}$
}

${ }^{1}$ Nuffield Department of Primary Care Health Sciences, University of Oxford, Oxford OX2 6GG, UK; ${ }^{2}$ Respiratory and Vaccine Preventable Bacteria Reference Unit, Public Health England, 61 Colindale Avenue, London NW9 5HT, UK; ${ }^{3}$ mmmunisation, Hepatitis and Blood Safety Department, Public Health England

\begin{abstract}
Objective To estimate the prevalence and clinical severity of whooping cough (pertussis) in school age children presenting with persistent cough in primary care since the introduction and implementation of the preschool pertussis booster vaccination.

Design Prospective cohort study (November 2010 to December 2012).

Setting General practices in Thames Valley, UK.

Participants 279 children aged 5 to 15 years who presented in primary care with a persistent cough of two to eight weeks' duration. Exclusion criteria were cough likely to be caused by a serious underlying medical condition, known immunodeficiency or immunocompromise, participation in another clinical research study, and preschool pertussis booster vaccination received less than one year previously.
\end{abstract}

Main outcome measures Evidence of recent pertussis infection based on an oral fluid anti-pertussis toxin $\lg G$ titre of at least 70 arbitrary units. Cough frequency was measured in six children with laboratory confirmed pertussis.

Results 56 (20\%, $95 \%$ confidence interval $16 \%$ to $25 \%$ ) children had evidence of recent pertussis infection, including 39 (18\%, $13 \%$ to $24 \%)$ of 215 children who had been fully vaccinated. The risk of pertussis was more than three times higher $(21 / 53 ; 40 \%, 26 \%$ to $54 \%)$ in children who had received the preschool pertussis booster vaccination seven years or more previously than in those who had received it less than seven years previously $(20 / 171 ; 12 \%, 7 \%$ to $17 \%)$. The risk of pertussis was similar between children who received five and three component preschool pertussis booster vaccines (risk ratio for five component vaccine $1.14,0.64$ to 2.03 ). Four of six children in whom cough frequency was measured coughed more than 400 times in 24 hours.
Conclusions Pertussis can still be found in a fifth of school age children who present in primary care with persistent cough and can cause clinically significant cough in fully vaccinated children. These findings will help to inform consideration of the need for an adolescent pertussis booster vaccination in the United Kingdom.

Study registration UK Clinical Research Network portfolio ID 8361.

\section{Introduction}

Whooping cough (pertussis) is one of the most common vaccine preventable diseases, causing nearly 300000 deaths a year in children worldwide. ${ }^{1}$ An accelerated schedule of primary pertussis vaccinations at 2, 3, and 4 months was introduced in the United Kingdom in 1990, and primary vaccine coverage has been more than $90 \%$ since $1992 .{ }^{2}$ However, immunity following vaccination is reported to last for only four to 12 years and immunity after infection for seven to 20 years. ${ }^{3}$ Pertussis in adolescents is recognised as a source of considerable socioeconomic burden. In the United States, pertussis was reported to be associated with a mean medical cost of $\$ 242$ (£144; €177) and to cause absence from school in $83 \%$ of adolescents for a mean of 5.5 days over a two year period. ${ }^{4}$

Between 2001 and 2005, evidence of recent pertussis infection was reported in $37 \%$ of UK school age children presenting with persistent cough in primary care. ${ }^{5}$ A preschool pertussis booster vaccination was introduced in the UK in October 2001. Current UK recommendations are that children should receive a preschool pertussis booster vaccination containing a three component (Infanrix-IPV) or five component (Repevax) acellular pertussis vaccine three years after completion of the primary vaccination course or soon afterwards. ${ }^{6}$ Compared with 
children who receive only a three dose course of primary vaccinations, the risk of pertussis is approximately halved in children who also receive the preschool pertussis booster vaccination (vaccine effectiveness $46 \%, 95 \%$ confidence interval $-7 \%$ to $71 \%$ ). ${ }^{2}$ However, an adolescent pertussis booster vaccination has so far not been introduced in the UK.

The introduction of an adolescent pertussis booster vaccination in several countries, including Australia, Canada, France, Germany, and the United States, was based on modelling of its potential cost effectiveness and observed increases in the incidence of pertussis among adolescents and adults..$^{7-9}$ In the United States, the adolescent pertussis booster vaccination (Tdap) has been only partially effective in preventing admission of infants to hospital due to pertussis ${ }^{10}$ possibly as a result of limited vaccination coverage. ${ }^{11}$ However, a sustained decreasing trend in the ratio of the incidence of pertussis in adolescents compared with other age groups has been observed since the introduction of the adolescent Tdap vaccination in 2005. ${ }^{12}$

A sustained increase in the incidence of pertussis among older children and adults, both before and since the introduction of the preschool pertussis booster vaccination, has been described in the UK. ${ }^{2}$ This is largely due to the availability of serology testing from 2002 onwards. No significant changes have been observed in pertussis related hospital admissions over the same period. From the last quarter of 2011, however, further increases in pertussis activity were observed in teenagers and older adults, which continued into 2012 and extended to all age groups, leading to a national outbreak being declared in April 2012. ${ }^{13}$ Although the incidence of pertussis fell in 2013, levels of disease continued to be raised in non-infant age groups compared with pre-2012 levels. $^{14}$

Although the main aim of pertussis vaccination is to reduce the risk of severe pertussis during infancy, the World Health Organization recommends that decisions concerning the introduction of national adolescent booster vaccination programmes should be based on the likelihood of these being cost effective and reducing the incidence of pertussis in the target age group, as the evidence is still insufficient to support their introduction to reduce severe pertussis in infants. ${ }^{9}$

Economic evaluations conducted to date suggest that vaccination of adolescents may be cost effective, but the robustness of these findings is limited by a high degree of uncertainty regarding key parameters, including the incidence of symptomatic and asymptomatic cases of pertussis and the costs associated with clinical pertussis in different age groups. ${ }^{15}$ The Joint Committee on Vaccination and Immunisation is reviewing the need for an adolescent pertussis booster vaccination in the UK and has established the need for further research to estimate the likely burden of disease in this age group. ${ }^{16}$ This study aims to inform these discussions by estimating the prevalence and clinical severity of pertussis in UK school age children who present in primary care with persistent cough following the introduction and implementation of the preschool pertussis booster vaccination.

\section{Methods}

\section{Participants}

Between November 2010 and December 2012, we prospectively recruited children aged 5 to 15 years who presented in primary care with a persistent cough of two to eight weeks' duration. Post-infectious persistent cough (that is, persistent cough triggered by an acute respiratory tract infection) is typically described as lasting between three and eight weeks. ${ }^{17}$ However, we set the lower limit of our inclusion criterion for duration of cough at two weeks on the basis of WHO's clinical case definition of pertussis - that is, cough lasting two weeks or longer, accompanied by whooping, vomiting, and/or paroxysms of coughing, without other apparent cause. ${ }^{18}$

We recruited children from 22 general practices in Thames Valley, UK. Exclusion criteria included cough likely to be caused by a serious underlying medical condition (for example, cystic fibrosis, bronchiectasis, cardiac failure), known immunodeficiency or immunocompromise, and current participation in another clinical research study. To avoid confounding of raised oral fluid anti-pertussis toxin IgG titres by recent preschool pertussis booster vaccine administration, we also excluded children who received the preschool pertussis booster vaccination less than one year before presentation in primary care. We included children with asthma if their clinician diagnosed their persistent cough as post-infectious rather than asthma related.

\section{Data collection}

Healthcare professionals at participating general practices obtained written informed consent from a parent or guardian for each study participant. On study entry, data were recorded for each participant on date of birth, sex, duration of cough, and household smoking status. Data were extracted from participants' medical records on vaccinations and previous diagnoses of asthma and allergic rhinitis.

To assess the clinical severity of cough in children with laboratory confirmed pertussis, we did 24 hour cough monitoring using the Leicester Cough Monitor in pertussis positive children whose parents or guardians gave additional written informed consent. ${ }^{19}$ Cough frequency measured using the Leicester Cough Monitor has been validated against manual cough counts and subjective cough outcome measures, including cough visual analogue scales and the Leicester Cough Questionnaire. ${ }^{19-21}$

\section{Laboratory diagnosis of pertussis}

We obtained an oral fluid sample from each study participant on study entry. Oral fluid samples were sent to the Health Protection Agency, Colindale, London (now Public Health England) for analysis using an IgG antigen-capture enzyme-linked immunosorbent assay to detect anti-pertussis toxin $\mathrm{IgG} .{ }^{22}$ Recent pertussis infection was diagnosed in children with an anti-pertussis toxin $\mathrm{IgG}$ titre of at least 70 arbitrary units $(\mathrm{aU})$ in oral fluid. Oral fluid anti-pertussis toxin IgG titres between 60 and $69 \mathrm{aU}$ were considered to be borderline/elevated. Throat swabs were also obtained from study participants to detect Mycoplasma pneumoniae (data reported in a separate article).

\section{Sample size and statistical analysis}

To allow a $20 \%$ prevalence of pertussis in the study population to be estimated with a precision of plus or minus $5 \%,{ }^{23}{ }^{24}$ the target sample size was 300 patients, allowing for a $20 \%$ failure rate in obtaining oral fluid samples with sufficient total $\mathrm{IgG}$ for analysis. We summarised participants' baseline characteristics by using numbers and percentages for categorical variables and means and $95 \%$ confidence intervals for continuous variables.

We estimated the prevalence, with $95 \%$ confidence intervals, of laboratory confirmed pertussis in our study population of children presenting in primary care with persistent cough in all participants whose oral fluid samples contained sufficient total $\mathrm{IgG}$ for analysis. We also estimated the prevalence of pertussis in subgroups who had and had not received the preschool pertussis booster vaccination and who had received complete 
primary and preschool booster vaccinations. For each quarter of 2011 and 2012, we summarised the percentages (with 95\% confidence intervals) of children with laboratory confirmed pertussis among study participants and children aged 5 to 15 years in England and Wales whose samples were submitted to Public Health England for pertussis serology. We also summarised the percentages (with $95 \%$ confidence intervals) of study participants with laboratory confirmed pertussis one to three, three to five, five to seven, and seven or more years after receiving the preschool pertussis booster vaccination.

We calculated risk ratios with $95 \%$ confidence intervals in relation to a laboratory diagnosis of pertussis for age, sex, household smoking status, asthma, and complete primary pertussis vaccinations (that is, three doses of primary pertussis vaccine). We also calculated risk ratios with $95 \%$ confidence intervals for type of preschool pertussis booster vaccination (five component versus three component acellular pertussis vaccine) and time since receiving the preschool pertussis booster vaccination. The last factor was analysed as a categorical variable, as was age. We excluded children with borderline/elevated oral fluid anti-pertussis toxin $\mathrm{IgG}$ titres from these analyses.

We did not calculate risk ratios for receipt of the primary acellular pertussis vaccine, meningococcus $\mathrm{C}$ vaccine, pneumococcal conjugate vaccine, or preschool pertussis booster vaccination because these were introduced only in September 2004, November 1999, September 2006, and October 2001, respectively, and would therefore not have been made available to all participants. Additionally, we could not reliably define subgroups to whom these vaccinations would have been made available owing to uncertainties relating to vaccine supply, administration of catch-up vaccinations, and other factors relating to the implementation of changes in vaccination policy at different practices.

We presented daytime (0800 to 2200) and night time frequencies of cough in children with laboratory confirmed pertussis whose parents or guardians gave additional written informed consent for 24 hour cough monitoring. We used SPSS Statistics version 20 for analyses.

\section{Results}

We gained consent to obtain oral fluid samples from 302 children. Oral fluid samples from 15 children had insufficient total IgG for analysis, and eight children had received the preschool pertussis booster vaccination less than one year before presentation with persistent cough. In total, we included 279 (92\%) children in our study. Of these, 261 (94\%) children had received complete primary pertussis vaccinations (three doses in total of whole cell or acellular pertussis vaccine), of whom $155(59 \%)$ received three doses of whole cell pertussis vaccine; $224(80 \%)$ children had received the preschool pertussis booster vaccination, of whom 215 (96\%) had also received complete primary pertussis vaccinations; $135(60 \%)$ children had received a preschool pertussis booster vaccination containing a five component acellular pertussis booster vaccine.

Fifty six (20\%, 95\% confidence interval $16 \%$ to $25 \%$ ) children had evidence of recent pertussis infection. Figure $1 \Downarrow$ summarises the age distribution of children with laboratory confirmed pertussis. Oral fluid anti-pertussis toxin IgG titres in children with laboratory confirmed pertussis ranged from 75 to more than $1000 \mathrm{aU}$ (median $297.5 \mathrm{aU}$ ). Variations in quarterly pertussis positivity rates among study participants during 2011 and 2012 closely mirrored those observed nationally (fig $2 \Downarrow$ ). Forty one (18\%, $13 \%$ to $24 \%)$ of the 224 children who had received the preschool pertussis booster vaccination and 15 ( $27 \%, 16 \%$ to $41 \%$ ) of the 55 children who had not received the preschool pertussis booster vaccination had laboratory confirmed pertussis. Thirty nine $(18 \%, 13 \%$ to $24 \%)$ of the 215 children who had received complete primary pertussis vaccinations and the preschool pertussis booster vaccination had laboratory confirmed pertussis. Six children had borderline/elevated oral fluid anti-pertussis toxin IgG titres and are not considered in table $1 \Downarrow$.

Table $1 \Downarrow$ summarises the baseline characteristics of pertussis positive and pertussis negative study participants. Thirty five participants had a previous diagnosis of asthma (pertussis positive, $n=7$; pertussis negative, $n=28$ ). Only one participant (pertussis negative) had a previous diagnosis of allergic rhinitis. The risk of laboratory confirmed pertussis was more than four times higher in children who had received the preschool pertussis booster vaccination seven or more years previously (risk ratio 4.23, 95\% confidence interval 1.84 to 9.70 ) than in children who had received the booster vaccination one to three years previously (reference category). However, the risk of laboratory confirmed pertussis was similar between children who had received a preschool booster vaccination containing a five component versus a three component acellular pertussis vaccine (relative risk 1.14, 0.64 to 2.03). Increasing age and time since receiving the preschool pertussis booster vaccination were not independently associated with increased risk of pertussis. In a model that included both these factors, neither was statistically significant.

Figure $3 \Downarrow$ summarises the percentages of study participants diagnosed as having laboratory confirmed pertussis at different time intervals after receiving the preschool pertussis booster vaccination. The risk of laboratory confirmed pertussis increased sharply from $15 \%$ (7/46 children; $95 \%$ confidence interval $6 \%$ to $29 \%$ ) in children who had received the preschool pertussis booster vaccination five to seven years previously to $40 \%$ (21/53 children; $26 \%$ to $54 \%$ ) in children who had received the booster vaccination seven or more years previously. This last group had a more than threefold greater risk of pertussis compared with the overall risk of pertussis in children who had received the preschool pertussis booster vaccination less than seven years before presenting in primary care with persistent cough (20/171 children; $12 \%, 7 \%$ to $17 \%$ ).

Figure $4 \Downarrow$ summarises 24 hour cough frequencies in six children with laboratory confirmed pertussis between six and 16 weeks after their cough started. Four children coughed more than 400 times in 24 hours (participants 3, 4, 5, and 6). All six children had received complete primary and preschool booster vaccinations.

\section{Discussion}

Pertussis can still be found in a fifth of UK school age children who present in primary care with persistent cough, despite more than $90 \%$ coverage with complete primary vaccinations and approximately $80 \%$ coverage with the preschool booster vaccination. The risk of pertussis was more than threefold higher in children given the preschool pertussis booster vaccination more than seven years before presentation in primary care with persistent cough, compared with those given the booster less than seven years before presentation. Pertussis can still cause clinically significant cough in children who have received complete primary pertussis vaccinations and the preschool pertussis booster vaccination. 


\section{Comparison with previous research}

Although the prevalence of pertussis in this cohort was around half that previously reported in a cohort of children recruited before the preschool pertussis booster vaccination had been implemented (37\%), ${ }^{5}$ our findings show that pertussis is still prevalent among school age children who present in primary care with persistent cough. Detection of pertussis in our study population may have been enhanced by recruitment from April 2012 to December 2012, which coincided with a national pertussis epidemic. ${ }^{13}$ However, our estimate was similar to that reported by a prospective case series study by Philipson et al in New Zealand, which found anti-pertussis toxin IgG titres in oral fluid consistent with recent pertussis infection in $18 \%(12 / 66)$ of school age children presenting in primary care with persistent cough. $^{25}$

An adolescent pertussis booster vaccination was introduced in New Zealand in 2006. ${ }^{26}$ However, Philipson et al did not report data on coverage with the adolescent pertussis booster vaccination in their study population. ${ }^{25}$ Philipson et al also recruited participants over a much shorter period (May to October 2011) than we did in our study (November 2010 to December 2012), ${ }^{25}$ although a two year prospective study in the Netherlands reported a similar prevalence of pertussis confirmed by serology or polymerase chain reaction among children referred with persistent cough to an outpatient clinic (23/135 children; $17 \%){ }^{23}$

Recent reports from the United States found an increasing likelihood of pertussis after completion of a five dose series of acellular pertussis vaccinations (DTaP) given at the ages of 2 , 4,6 , and 15-18 months and 4-6 years. In a large cohort analysis of fully vaccinated children, risk ratios for pertussis after the fifth dose of DTaP (using year 1 as the reference group) increased from 1.9 (95\% confidence interval 1.3 to 2.9 ) in year 2 to 8.9 (6.0 to 13.0 ) in year $6 .{ }^{27}$ Compared with an unvaccinated reference group, odds ratios for pertussis increased from 0.02 (0.01 to 0.04$)$ during the first year to $0.29(0.15$ to 0.54$)$ five years or more after receipt of a complete course of DTaP vaccinations. ${ }^{28}$ The odds of pertussis have previously been reported to increase by an average of $42 \%$ a year during the first five years. ${ }^{29}$ Our findings show a subsequent sharp increase in risk of pertussis, which more than triples after seven years.

Recent data from the United States have shown that previously vaccinated pertussis positive children and adolescents were less likely to develop severe illness (pneumonia or hospital admission) and had a shorter duration of cough than unvaccinated pertussis cases. ${ }^{30}$ However, our cough frequency results show that pertussis can still manifest as clinically significant cough in fully vaccinated children, even 16 weeks after the initial onset of cough.

\section{Strengths and limitations of study}

To our knowledge, this study represents the largest prospectively recruited cohort to date of school age children presenting with persistent cough in primary care. Oral fluid samples from more than $90 \%$ of children were suitable for analysis, and we used oral fluid anti-pertussis toxin IgG titres of $70 \mathrm{aU}$ or above to indicate evidence of recent pertussis infection. This threshold was derived from extensive validation against the standard serum anti-pertussis toxin IgG assay used in the UK Pertussis National Reference Laboratory and showed a sensitivity of $93 \%$ and a specificity of $94 \% .{ }^{22}$ Raised serum anti-pertussis toxin IgG titres have been shown to be positive in less than $1 \%$ of the general population, ${ }^{31}$ so they are a reliable indicator of recent pertussis infection. However, obtaining oral fluid samples is less invasive than obtaining blood samples. We considered this to be particularly advantageous in the context of our study, given that we were recruiting children.

The similarities we observed between temporal variations in pertussis positivity rates in our study cohort and corresponding data from England and Wales show that our findings are likely to be generalisable on a national scale. These similarities also indicate that, although diagnosis of pertussis based on clinical features is imprecise, ${ }^{32} 33$ persistent cough, with or without other classic symptoms such as whooping and vomiting, is useful as a pragmatic clinical phenotype for identifying suspected cases of pertussis.

Nevertheless, some children who met the study eligibility criteria may not have been invited to take part in the study. We were unable to retrospectively estimate the total number of eligible children who presented during our recruitment period because of variations in coding of consultations for persistent cough at participating general practices. Keeping prospective records of every patient screened by every clinician was also not feasible because of the time constraints associated with consulting in a primary care setting. This is a known limitation of studies that recruit participants opportunistically from primary care settings during periods of heightened disease activity, ${ }^{34}$ including studies recruiting patients with persistent cough. ${ }^{25}$

However, recent studies that recruited participants with persistent cough and used the same oral fluid anti-pertussis toxin IgG assay that we used found that around $90 \%$ of eligible patients approached gave consent for study entry. ${ }^{25}{ }^{35}$ Based on audits conducted at six high recruiting general practices with on-site research staff, we estimated that $51 \%$ (197/390) of eligible children were recruited. This is broadly consistent with what we observed during the prospective cohort study that we conducted before the preschool pertussis booster vaccination had been implemented. ${ }^{5}$

Recruitment of study participants occurred in 22/27 (81\%) general practices that took part in our study. These practices were located across the whole Thames Valley region (Oxfordshire, Buckinghamshire, Berkshire, and Milton Keynes) and covered populations with a broad spectrum of socioeconomic deprivation (Index of Multiple Deprivation 2012 score range 5.9 to 23.2 ). ${ }^{36}$

To avoid confounding of raised oral fluid anti-pertussis toxin IgG titres by recent administration of the preschool pertussis booster vaccination, we excluded children who had received a booster less than one year before presentation in primary care. This cut-off was based on standard laboratory practice during our recruitment period. However, recent mathematical modelling of oral fluid anti-pertussis toxin IgG titres measured up to 13 months after receipt of the preschool pertussis booster vaccination suggest that these may be confounded for as long as three years. ${ }^{22}$ Even so, our estimate of the prevalence of pertussis based on study participants who had not received the preschool pertussis booster vaccination or who had received their booster vaccination more than three years previously would have been similar (50/213 participants; $23 \%, 18 \%$ to $30 \%$ ).

Additionally, our study findings clearly show a sharp increase in the risk of pertussis seven years after receipt of the preschool pertussis booster vaccination. This observation may have been influenced by confounding factors, such as changes in age related exposure to pertussis and herd immunity.

We were unable to adjust for asthma, allergic rhinitis, or presence of household smokers because of their low prevalence among study participants. We were also unable to explore the possibility of vaccine interaction between the measles, mumps, 
and rubella (MMR) and pertussis booster vaccinations because only four pertussis positive and nine pertussis negative participants who had received the preschool pertussis booster vaccination did not receive the MMR booster vaccination. The lower proportion of pertussis positive participants who received the primary acellular pertussis vaccine most likely reflects the increased risk of pertussis in older children, some of whom would have received their primary pertussis vaccinations before the acellular pertussis vaccine had been introduced.

We gained consent to do 24 hour cough monitoring only in a convenience sample of six pertussis positive children because a research nurse was available to do cough monitoring only during the final six months of the study. Clinical severity of cough in children with pertussis has previously been reported only with unvalidated subjective measures, such as parent reported number of cough episodes and cough related sleep disturbance. ${ }^{5}$ Future research should consider the use of less resource intensive methods, including four hour cough monitoring and validated, parent specific, cough related quality of life measures. ${ }^{20} 37$

\section{Implications for vaccination policy}

Our findings show that a significant burden of illness associated with pertussis remains among school age children who present in UK primary care with persistent cough, even after implementation of the preschool pertussis booster vaccination and despite high levels of vaccination coverage. Pertussis is still prevalent among school age children who present with persistent cough in primary care and can still manifest as clinically significant cough in fully vaccinated children. Furthermore, the risk of pertussis more than triples in children who received the preschool pertussis booster vaccination seven years or longer before presenting in primary care with persistent cough. As burden of illness has been identified as a key area in which data are needed to inform modelling of the likely cost effectiveness of a national adolescent pertussis booster programme, ${ }^{15}$ these findings will help to inform consideration of the need for an adolescent pertussis booster vaccination in the UK.

To determine whether an adolescent pertussis booster vaccination would be cost effective, further research is needed to examine the epidemiology and socioeconomic burden of pertussis in adolescents and adults and to develop dynamic economic evaluation models based on national data. If an adolescent pertussis booster vaccination is found to be cost effective, administering this alongside the routine meningococcus $\mathrm{C}$ and tetanus, diphtheria, and inactivated polio adolescent booster vaccinations, currently recommended at around 14 years of age, ${ }^{6}$ may be the most efficient way of ensuring high vaccination coverage.

We acknowledge the support of the National Institute for Health Research (NIHR), through the Primary Care Research Network. This study was adopted by the University of Oxford Primary Care Clinical Trials Unit. We thank Christy Toms, Tricia Carver, Louise Jones, and Maria Breen for all their hard work in supporting the set-up and day to day management of the study. We also thank Brendan Bradley and David Judge for their support with IT and data management. We thank Surinder Birring, Sergio Matos, and Kai Lee for their assistance with setting up the Leicester Cough Monitor and Marie-Lucie Gibbons for assistance with obtaining cough recordings. We also thank staff at the Public Health England Respiratory and Vaccine Preventable Bacteria Reference Unit for assistance with processing of oral fluid specimens. Finally, we thank all the healthcare professionals, general practice staff, and patients who participated in our study.
Contributors: KW, NKF, TGH, and AH contributed to the development of the protocol. This work was part of KW's doctoral thesis supervised by $\mathrm{AH}$ and DM. KW led day to day management of the study and was chief investigator for the study. $\mathrm{HC}$ provided data on pertussis detection rates in England and Wales during 2011 and 2012. KW did all other data analyses. Rafael Perera (University of Oxford) and Nick Andrews (Public Health England) provided statistical advice. NKF and TGH were responsible for processing oral fluid samples and reporting their results. $\mathrm{KW}$ wrote the first draft of the report. All authors contributed comments on and edits to the report. KW and $\mathrm{AH}$ are the guarantors.

Funding: This paper presents independent research funded by the National Institute for Health Research (NIHR) School for Primary Care Research. The views expressed are those of the authors and not necessarily those of the NHS, the NIHR, or the Department of Health. The cough monitor component of the study was funded by an Oxfordshire Health Services Research Committee grant. The funding sources were not involved in study design; data collection, analysis, or interpretation; report writing; or submission. The views expressed are those of the authors and not necessarily those of the NHS, the NIHR, or the Department of Health.

Competing interests: All authors have completed the ICMJE uniform disclosure form at www.icmje.org/coi_disclosure.pdf (available on request from the corresponding author) and declare: KW held an NIHR doctoral research fellowship; $\mathrm{AH}$ is Chairman of the Joint Committee on Vaccination and Immunisation Adolescent Sub-committee; $\mathrm{HC}$ and GA are members of the Public Health England Immunisation Department, which has provided vaccine manufacturers with post-marketing surveillance reports, which these companies are required to submit to the UK Licensing Authority in compliance with their risk management strategy; a cost recovery charge is made for these reports; no reports relating to vaccines containing pertussis have been provided to date.

Ethical approval: This study was approved by Berkshire Research Ethics Committee (reference 10/H0505/44). A parent or guardian gave written informed consent for each participant.

Transparency: The guarantors affirm that the manuscript is an honest, accurate, and transparent account of the study being reported; that no important aspects of the study have been omitted; and that any discrepancies from the study as planned and registered have been explained.

Data sharing: The corresponding author should be contacted regarding requests for the study protocol or unpublished data.

Crowcroft NS, Pebody RG. Recent developments in pertussis. Lancet 2006;367:1926-36. Campbell H, Amirthalingam G, Andrews N, Fry NK, George RC, Harrison TG, et al. Accelerating control of pertussis in England and Wales. Emerg Infect Dis 2012;18:38-47. Wendelboe AM, Van Rie A, Salmaso S, Englund JA. Duration of immunity against pertussis after natural infection or vaccination. Pediatr Infect Dis J 2005;24(5 suppl):S58-61.

4 Lee GM, Lett S, Schauer S, LeBaron C, Murphy TV, Rusinak D, et al. Societal costs and morbidity of pertussis in adolescents and adults. Clin Infect Dis 2004;39:1572-80.

5 Harnden A, Grant C, Harrison T, Perera R, Brueggemann AB, Mayon-White R, et al. Whooping cough in school age children with persistent cough: prospective cohort study in primary care. BMJ 2006;333:174-7.

6 NHS Immunisation Information. The complete routine immunisation schedule 2013/14 www.gov.uk/government/uploads/system/uploads/attachment_data/file/227651/8515_ DoH_Complete_Imm_schedule_A4_2013_09.pdf.

7 Lee GM, LeBaron C, Murphy TV, Lett S, Schauer S, Lieu TA. Pertussis in adolescents and adults: should we vaccinate? Pediatrics 2005;115:1675-84

8 Caro JJ, Getsios D, El-Hadi W, Payne K, O'Brien JA. Pertussis immunization of adolescents in the United States: an economic evaluation. Pediatr Infect Dis J 2005;24(5 suppl):S75-82.

9 Pertussis vaccines: WHO position paper. Wkly Epidemiol Rec 2010;40:385-400.

10 Auger KA, Patrick SW, Davis MM. Infant hospitalizations for pertussis before and after Tdap recommendations for adolescents. Pediatrics 2013;132:e1149-55.

11 Clark T. Pertussis epidemiology and vaccination in the United States and the Latin American Pertussis Project. www.who.int/immunization/sage/meetings/2012/november/ 7 Pertussis -Outbreaks in USA-Tomas_Clark.pdf.

12 Skoff TH, Cohn AC, Clark TA, Messonnier NE, Martin SW. Early impact of the US Tdap vaccination program on pertussis trends. Arch Pediatr Adolesc Med 2012;166:344-9.

13 Public Health England. Pertussis in England and Wales: fourth quarter 2012 data and consolidated annual report for 2012. Health Protection Report 2013;7(14-17) (available at www.hpa.org.uk/hpr/archives/2013/hpr14-1713.pdf). 


\section{What is already known on this topic}

Before the introduction of a preschool pertussis booster vaccination in 2001, whooping cough (pertussis) could be found in $37 \%$ of school age children presenting in UK primary care with persistent cough

An adolescent pertussis booster vaccination has so far not been introduced in the UK

\section{What this study adds}

Pertussis can still be found in a fifth of school age children who present in primary care with persistent cough and can cause clinically significant cough in fully vaccinated children

The risk of pertussis is more than threefold higher in children who received the preschool pertussis booster vaccination seven years or longer before presenting in primary care with persistent cough compared with those given the booster more recently

These findings will help to inform consideration of the need for an adolescent pertussis booster vaccination in the UK

14 Public Health England. Laboratory confirmed pertussis in England: data to end-December 2013. Health Protection Report 2014;8(5-6) (available at www.hpa.org.uk/hpr/archives/ 2014/hpr05-0614.pdf)

15 Rivero-Santana A, Cuellar-Pompa L, Sanchez-Gomez LM, Perestelo-Perez L, Serrano-Aquilar $P$. Effectiveness and cost-effectiveness of different immunization strategies against whooping cough to reduce child morbidity and mortality. Health Policy 2014;115:82-91.

16 Joint Committee on Vaccination and Immunisation Sub-committee on adolescent vaccinations. Minutes of meeting held on Friday 27 January 2012. www.gov.uk/government/ uploads/system/uploads/attachment_data/file/158008/JCVI-Minutes-of-sub-committeeon-adolescent-vaccinations-27-January-2012.pdf.

17 Kwon N-H, Oh M-J, Min T-H, Lee B-J, Choi D-C. Causes and clinical features of subacute cough. Chest 2006;129:1142-7.

18 Cherry JD, Tan T, Wirsing von König CH, Forsyth KD, Thisyakorn U, Greenberg D, et al. Clinical definitions of pertussis: summary of a global pertussis initiative roundtable meeting, February 2011. Clin Infect Dis 2012;54:1756-64.

19 Birring SS, Fleming T, Matos S, Raj AA, Evans DH, Pavord ID. The Leicester Cough Monitor: preliminary validation of an automated cough detection system in chronic cough. Eur Respir J 2008:31:1013-8.

20 Lee KK, Savani A, Matos S, Evans DH, Pavord ID, Birring SS. 4-hour cough frequency monitoring in chronic cough. Chest 2012;142:1237-43.

21 Lee KK, Matos S, Evans DH, White P, Pavord ID, Birring SS. A longitudinal assessment of acute cough. Am J Respir Crit Care Med 2013:187:991-7.

22 Fry NK, Litt DJ, Duncan J, Vaghji L, Warrener L, Samuel D, et al. Modelling anti-pertussis toxin IgG antibody decay following primary and preschool vaccination with an acellular pertussis vaccine in UK subjects using a modified oral fluid assay. $J$ Med Microbiol 2013;62:1281-9.

23 Versteegh FG, Weverling GJ, Peeters MF, Wilbrink B, Veenstra-van Schie MY, van Leeuwen-Gerritsen JM, et al. Community-acquired pathogens associated with prolonged coughing in children: a prospective cohort study. Clin Microbiol Infect 2005;11:801-7.

24 Senzilet LD, Halperin SA, Spika JS, Alagaratnam M, Morris A, Smith B, for the Sentinel Health Unit Surveillance System Pertussis Working Group. Pertussis is a frequent cause of prolonged cough illness in adults and adolescents. Clin Infect Dis 2001;32:1691-7.

25 Philipson K, Goodyear-Smith F, Grant CC, Chong A, Turner N, Stewart J. When is acute persistent cough in school-age children and adults whooping cough? A prospective case series study. Br J Gen Pract 2013;63:e573-9.

26 Grant CC, Reid S. Pertussis continues to put New Zealand's immunisation strategy to the test. N Z Med J 2010;123:46-61.
27 Tartof SY, Lewis M, Kenyon C, White K, Osborn A, Liko J, et al. Waning immunity to pertussis following 5 doses of DTaP. Pediatrics 2013;131:e1047-52.

28 Misegades LK, Winter K, Harriman K, Talarico J, Messonnier NE, Clark TA, et al. Association of childhood pertussis with receipt of 5 doses of pertussis vaccine by time since last vaccine dose, California, 2010. JAMA 2012;308:2126-32.

29 Klein NP, Bartlett J, Rowhani-Rahbar A, Fireman B, Baxter R. Waning protection after fifth dose of acellular pertussis vaccine in children. N Engl J Med 2012;367:1012-9.

30 Barlow RS, Reynolds LE, Cieslak PR, Sullivan AD. Vaccinated children and adolescents with pertussis infections have decreased illness severity and duration, Oregon 2010-2012. Clin Infect Dis 2014:58:1523-9.

31 De Melker HE, Versteegh FG, Conyn-Van Spaendonck MA, Elvers LH, Berbers GA, van $\operatorname{der}$ Zee $A$, et al. Specificity and sensitivity of high levels of immunoglobulin $G$ antibodies against pertussis toxin in a single serum sample for diagnosis of infection with Bordetella pertussis. J Clin Microbiol 2000;38:800-6.

32 Cornia PB, Hersh AL, Lipsky BA, Newman TB, Gonzales R. Does this coughing adolescent or adult patient have pertussis? JAMA 2010;304:890-6.

33 Wang K, Harnden A. Pertussis-induced cough. Pulm Pharmacol Ther 2011;24:304-7.

34 Little P, Hobbs FD, Moore M, Mant D, Williamson I, McNulty C, et al. Clinical score and rapid antigen detection test to guide antibiotic use for sore throats: randomised controlled trial of PRISM (primary care streptococcal management). BMJ 2013;347:55806.

35 Wang K, Birring SS, Taylor K, Fry NK, Hay AD, Moore M, et al. Montelukast for postinfectious cough in adults: a double-blind randomised placebo-controlled trial. Lancet Respir Med 2014;2:35-43.

36 Public Health England. National General Practice Profiles. 2013. http://fingertips.phe.org. uk/profile/general-practice.

37 Newcombe PA, Sheffield JK, Chang AB. Parent cough-specific quality of life: development and validation of a short form. J Allergy Clin Immunol 2012 131:1069-74.

Accepted: 13 May 2014

\section{Cite this as: BMJ 2014;348:g3668}

This is an Open Access article distributed in accordance with the Creative Commons Attribution Non Commercial (CC BY-NC 3.0) license, which permits others to distribute, remix, adapt, build upon this work non-commercially, and license their derivative works on different terms, provided the original work is properly cited and the use is non-commercial. See: http://creativecommons.org/licenses/by-nc/3.0/. 


\section{Table}

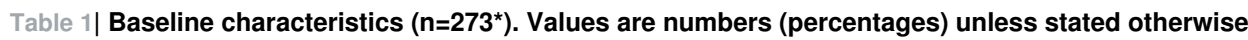

\begin{tabular}{|c|c|c|c|}
\hline Characteristic & Pertussis positive $(n=56)$ & Pertussis negative ( $\mathrm{n}=\mathbf{2 1 7}$ ) & Risk ratio $(95 \% \mathrm{Cl})$ \\
\hline Mean $(95 \% \mathrm{Cl})$ age, years & $11.0(5.1$ to 15.5$)$ & $9.3(5.1$ to 15.6$)$ & - \\
\hline \multicolumn{4}{|l|}{ Age group: } \\
\hline $5-9$ years & $17(30)$ & $132(61)$ & 1 \\
\hline $10-15$ years & $39(70)$ & $85(39)$ & 2.76 (1.64 to 4.62$)$ \\
\hline Mean $(95 \% \mathrm{Cl})$ time since PSB, years & $6.4(1.1$ to 10.3$)$ & $4.5(1.4$ to 9.2$)$ & - \\
\hline \multicolumn{4}{|l|}{ Time since PSB†: } \\
\hline $1-3$ years & $6(15)$ & $58(33)$ & 1 \\
\hline 3-5 years & $7(17)$ & $51(29)$ & 1.29 (0.46 to 3.61$)$ \\
\hline $5-7$ years & $7(17)$ & $37(21)$ & $1.70(0.61$ to 4.71$)$ \\
\hline$\geq 7$ years & $21(51)$ & $32(18)$ & $4.23(1.84$ to 9.70$)$ \\
\hline Mean $(95 \% \mathrm{Cl})$ duration of cough, weeks & $5.0(2.0$ to 8.0$)$ & $4.2(2.0$ to 8.0$)$ & - \\
\hline Male sex & $26(46)$ & $112(52)$ & 0.85 (0.53 to 1.35$)$ \\
\hline Smoker(s) in household & $6(11)$ & $44(20)$ & $0.54(0.24$ to 1.18$)$ \\
\hline Asthma & $7(13)$ & $28(13)$ & $0.97(0.48$ to 1.97$)$ \\
\hline \multicolumn{4}{|l|}{ Primary vaccinations: } \\
\hline Pertussis (complete vaccinations $\ddagger$ ) & $50(89)$ & $205(94)$ & 0.59 (0.29 to 1.18$)$ \\
\hline $\mathrm{aP \S}$ & $11 / 50(22)$ & $93 / 205(45)$ & $\S \S$ \\
\hline menC $\S^{\star \star}$ & $33 / 50(66)$ & 149/205 (73) & $\S \S$ \\
\hline PCV§t† & $7 / 50(14)$ & $71 / 205(35)$ & $\S \S$ \\
\hline \multicolumn{4}{|l|}{ Booster vaccinations: } \\
\hline PSB $\neq \ddagger$ & $41(73)$ & $178(82)$ & $\S \S$ \\
\hline PSB-5† & $26(63)$ & $106(60)$ & $1.14(0.64$ to 2.03$)$ \\
\hline MMR $\dagger$ & $37(90)$ & 169 (95) & ๆศ \\
\hline
\end{tabular}

$\mathrm{aP}=$ acellular pertussis vaccine; $\mathrm{menC}=$ meningococcus $\mathrm{C}$ vaccine; $\mathrm{PCV}=$ pneumococcal conjugate vaccine; $\mathrm{PSB}=$ preschool pertussis booster vaccination; PSB-5=preschool pertussis booster vaccination containing five component acellular pertussis vaccine; MMR=measles, mumps, and rubella vaccination. *Does not include 6 children with borderline/elevated oral fluid antipertussis toxin $\lg G$ titres.

†Percentages calculated using children who received preschool pertussis booster vaccination as denominator (pertussis positive, $n=41$; pertussis negative, $n=178$ ). $\ddagger$ Three doses in total of whole cell or acellular pertussis vaccine.

§At least 1 dose of vaccine in children who also received complete primary pertussis vaccinations.

IIntroduced in UK in September 2004 and temporarily administered during 2000-01.

${ }^{* *}$ Introduced in UK in November 1999.

††Introduced in UK in September 2006.

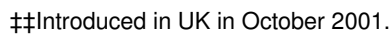

$\S \S$ Risk ratios were not calculated for these vaccinations because they would not have been available to all participants, and subgroups to whom they would have been made available could not be reliably defined owing to uncertainties regarding vaccine supply, administration of catch-up vaccinations, and other factors relating to implementation of vaccination policy changes at different practices.

IIRRisk ratio not calculated because only small number of participants received preschool pertussis booster vaccination without MMR booster vaccination (pertussis positive, $n=4$; pertussis negative, $n=9$ ). 


\section{Figures}

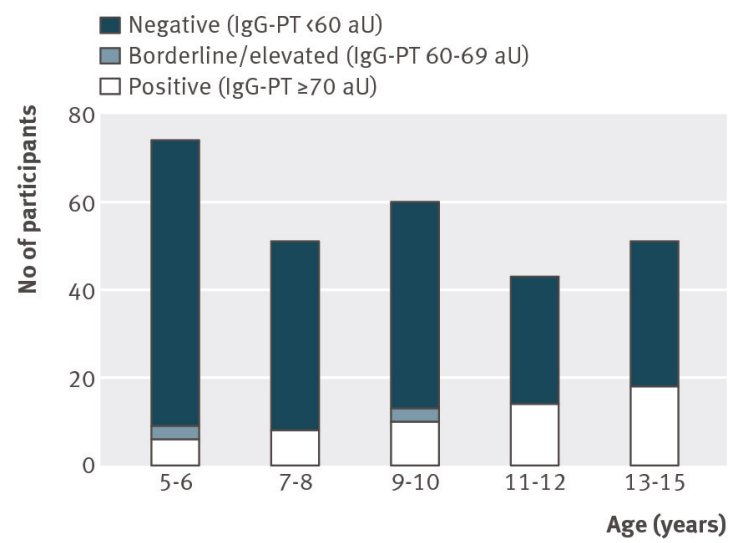

Fig 1 Age distribution of laboratory confirmed pertussis cases. aU=arbitrary units; IgG-PT=oral fluid anti-pertussis toxin lgG titre. Laboratory confirmed pertussis was diagnosed in children with positive IgG-PT titre ( $\geq 70$ aU). *Children born before October 1997 are unlikely to have been offered the preschool pertussis booster vaccination, as this was introduced only in October 2001

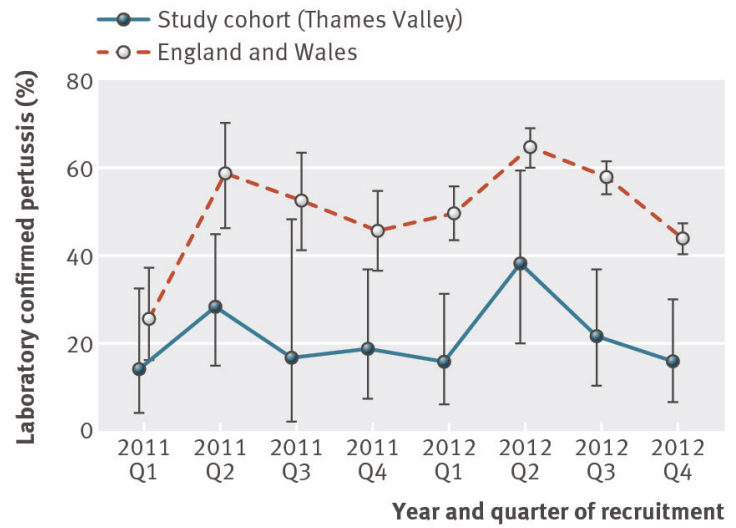

Fig 2 Percentage of children aged 5 to 15 years with laboratory confirmed pertussis per quarter (2011 to 2012), with 95\% confidence intervals. Study cohort (Thames Valley): laboratory confirmed pertussis diagnosed by oral fluid anti-pertussis toxin IgG titre $\geq 70$ arbitrary units; percentage of children with laboratory confirmed pertussis calculated on basis of all oral fluid samples containing sufficient total lgG for analysis. England and Wales: laboratory confirmed pertussis diagnosed by serology; percentage of children with laboratory confirmed pertussis calculated on basis of all samples submitted to Public Health England (formerly Health Protection Agency) for analysis

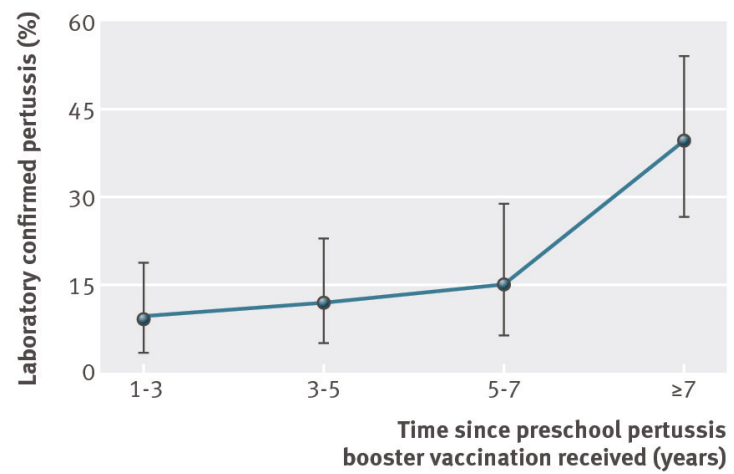

Fig 3 Laboratory confirmed pertussis in children presenting with persistent cough in primary care after receiving preschool pertussis booster vaccination $(n=224)$. Error bars represent $95 \%$ confidence intervals 


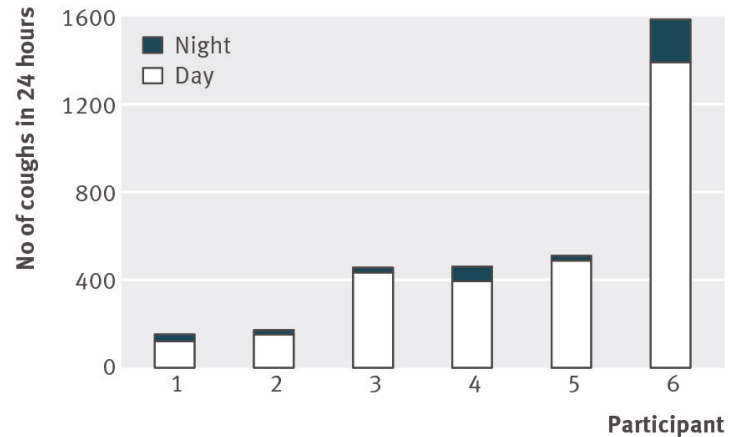

Fig 424 hour cough frequency in children with laboratory confirmed pertussis $(n=6)$. Daytime cough monitoring period was from 0800 to 2200 . All six participants received complete primary pertussis vaccinations and preschool pertussis booster vaccination 